\title{
Language Technology and Computational Linguistics
}

\author{
Puspanjali Hazarika, Md. Mostafa Rashel
}

\begin{abstract}
In the present world, information technology is the predominant part, which has the ability to change the future of this Globe. Being a Futuristic Technology. Computational linguistics can change communication models among human beings. Due to the changing context and development of Natural Language Processing various new doors are open in the fields of computational linguistics. Computational Linguistics (CL) increases the applicability of Language Technology towardsman-machine interactions. Globalization can convert the world into a small village. For interchange human knowledge among various communities, Auto language processing play a vital role. In this paper, it is tried to discuss the various dimension of language technology and computational linguistics.
\end{abstract}

Keywords: Language Technology, Natural Language Processing, Machine Translation Computational linguistics, Humanlanguage.

\section{INTRODUCTION}

Language is a milestone for communication among human beings. Language technology denotes the integration of Computer Science and the phenomenon of linguistics. Computational linguistics (CL) and Natural Language Processing (NLP) deal with how human language transforms into a computer program.

Language Technology accomplishes with computational methods, programs, and various electronic devices. Electronic devices work on speech recognition, manufacturing, and adjusting the text. Everyday develops dimension of Language technology. In regional language, it plays a significant role in communication and preservation and promoting specificity.

\section{LANGUAGE TECHNOLOGY'S GOALS AND POSSIBILITIES}

The primary objectives and possibilities of language technologies are as follows(Rashel 2011: 179):

- To improve the communication between humans and machinesmost easily.

- To increase the productivity of any language in a machine-readable format.

- Language technology increases virtual communication in text and speech forms.

Revised Manuscript Received on November 06, 2019.

* Correspondence Author

Puspanjali Hazarika*, Research Scholar of Assamese Language

Md. Mostafa Rashel, Assistant Professor of Linguistics, Department of English, Daffodil International University, Bangladesh.

(c) The Authors. Published by Blue Eyes Intelligence Engineering and Sciences Publication (BEIESP). This is an open access article under the CC-BY-NC-ND license http://creativecommons.org/licenses/by-nc-nd/4.0/
- Language Technology plays a vital role in the preservation of endangered languages.

- Speech and text technology increase acceptability in communication.

- Text recognition provides a platform for heritage script. etc.

\section{FUNCTIONS OF LANGUAGE TECHNOLOGY}

In the context of the information society, Langue Technology plays a vital role in communication. The major aim of language technology is to create software tools that can process human language. Language technology has a significant position in Information Retrieval (IR). The weight of language in an information retrieval system depends on recall and precision rate. Language technology controls the following system(Uszkoreit 1997:3-4,Ledeneva\&Sidorov 2010:3, Rashel 2011:180):

- Data Fusion and Text Data Mining

- Information Extraction

- Question Answering

- Report Generation

- Speech Recognition

- Speech Synthesis

- Spoken Dialogue Systems

- Text Categorization

- Text Indexing

- Text Retrieval

- Text Summarization

- Translation Technologies

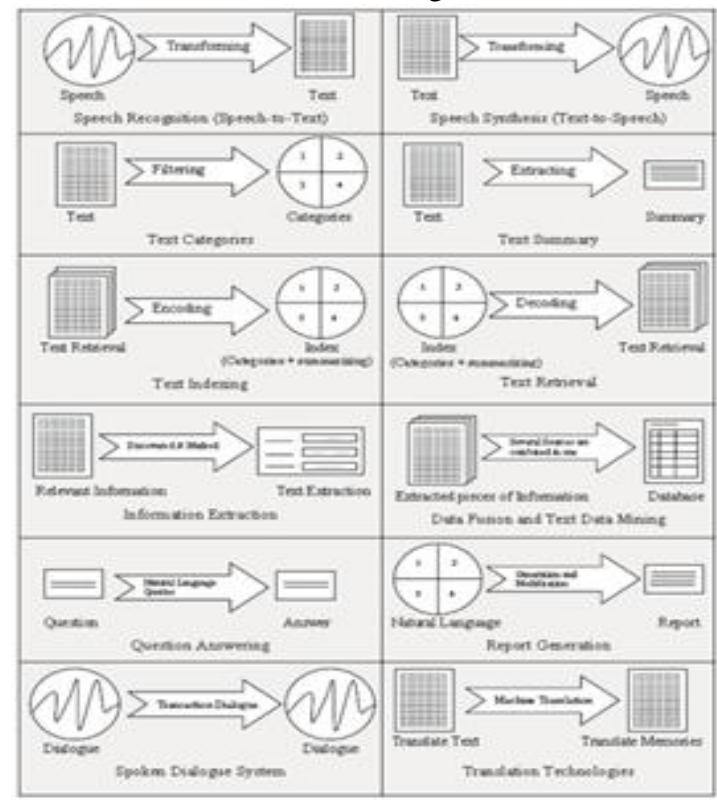

Figure- 1: Functions of Language Technology(Rashel 2011: 184)

Published By:

Blue Eyes Intelligence Engineering DOI: 10.35940/ijitee.A3877.119119

\& Sciences Publication (BEIESP) 


\section{Language Technology and Computational Linguistics}

\section{COMPUTATIONAL LINGUISTICS}

Computer technology can cover all significant parts of society. Computational Linguistics is an entirely virgin discipline in combination with Linguistics and Computer Science. Everyday change, the dimension of Computational Linguistics and add with the various hybrid area. The application of computer technology and linguistics theory can open a new milestones for communication for visually challenged persons.

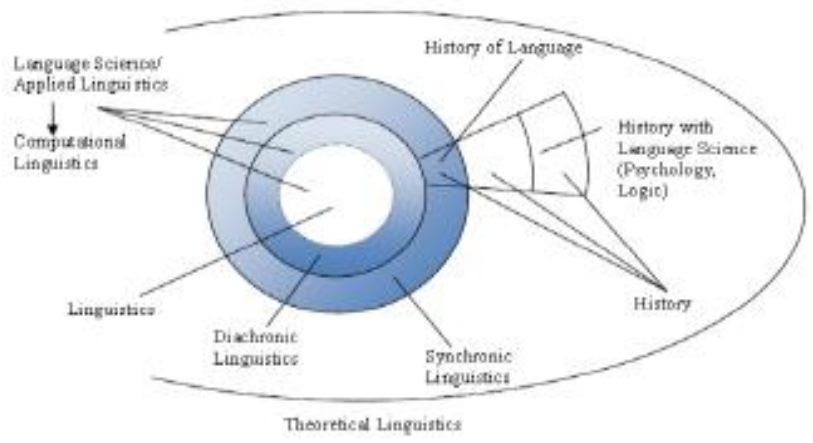

Figure-2: Position of Computational Linguistics in Linguistics Diversity (Rashel 2011: 185)

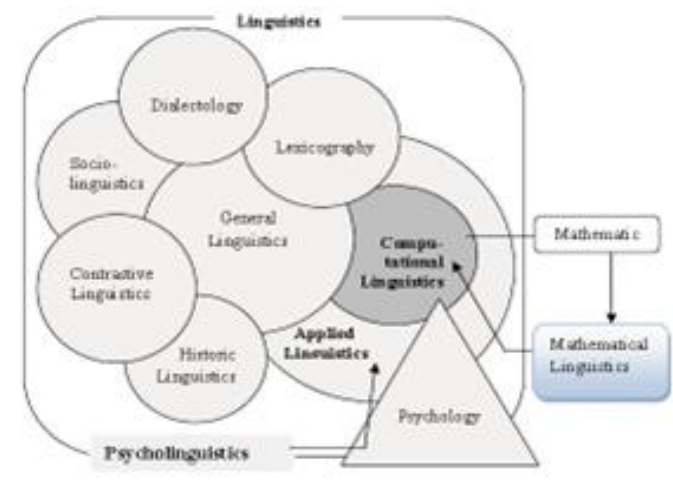

Figure- 3: Structural Relationship between Linguistics Science \& Computational Linguistics

(Bolshakov\& Gelbukh, 2004, Cited in Rashel 2011: 185)

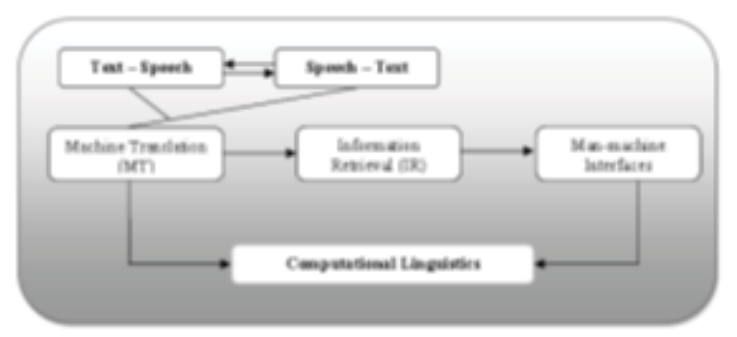

Figure: 4- Central Development of Computational Linguistics (Rashel 2011: 186)

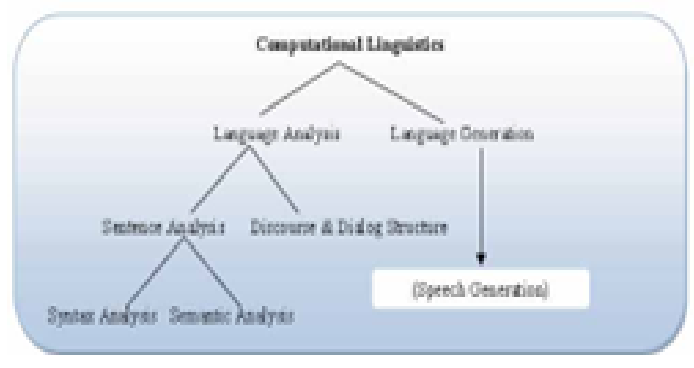

Figure-5: Structural Diagram of Computational Linguistics (Rashel 2011:186)
Now computational linguistics combined with various disciplines for solving communication problems such as mathematics, theoretical linguistics, engineering, etc., using Machine Translation has developed various problems such as auto-grammar checker, Extention vocabulary, and identification of parts of speech. MT provides translational capabilities with minimum time.

Speech Recognition(SR)is an integral part of Computational Linguistics, which has a significant demand among common people. Various Speech Recognition software has came into the software market. Using speech recognition technology,the various task becomes easy. Such as Information Retrieval (IR). Speech Recognition (SR) is another integral part of computational linguistics; SR provides an extensive learning and communication platform. Speech Generation(SG) solved the problems of natural sound reading of the written text (Rashel 2011: 180).

\section{OBJECTIVES OF COMPUTATIONAL LINGUISTICS}

The primaryobjectives of computational linguistics are as follows-

- Machine translation provides an opportunity for quick translation.

- Prepared a specific applied system for NL.

- The primary application area of Computational linguistics is- Information Retrieval (IR), Machine Translation, and Man-Machine Interaction(Grishman, 1994 cited from Rashel 2011: 181).

- These also combine with the Psychological process of Language Processing, Knowledge illustration, and Understanding.

The major application of computational linguistics are as follows-

- In the software market, every day increases the job opportunity for developing a search engine, IR models, etc.

- Increase effective communication among human beings.

- Increasing the efficiency in human-machine interaction.

- Remove the difficulties faced in modern communication.

- Save time for Second language learners using- MT, NLI, IR Grammar Checker, Style Chacker, etc.(Rashel 2011: 181)

- Various research groups work in AI using Computational linguistics.

\section{CONCLUSION}

The present world is run with ICT; without ICT, the world's community cannot survive in this environment. Due to the changing situation, Linguistics fields have been drastically changed and integrated with computer science, and various productive giftswere provided for society. AI, NLP, IR, SR, SG, etc., become integral tohuman society.

Published By:

Blue Eyes Intelligence Engineering 
Developing all languages in a complete computerization form is necessaryfor the application and preservation point of view. Developing a language into a computational form needs an expert professional with knowledge of Computer Science and linguistics.Developing a language and expandingit into a computational format is essential for that particular region. In the context of globalization, it is not easy to protect a language from code-mixing and code-switching; it istime to develop all languages to computational forms and provide access opportunities for common people in the communication medium.Applying computational linguistics is a significant task to protect the regional and local languages.

\section{REFERENCES}

1. Bolshakov, I. A., \&Gelbukh, A. (2004, June). Synonymous paraphrasing using wordnet and internet. In International Conference on Application of Natural Language to Information Systems (pp. 312-323). Springer, Berlin, Heidelberg. Retrieved from: DOI: https://doi.org/10.1007/978-3-540-27779-8_27

2. Cunningham, H., Gaizauskas, R. J., \& Wilks, Y. (1995). A general architecture for text engineering (GATE): A new approach to language engineering $R \& D$. University of Sheffield, Department of Computer Science. Retrieved from: https://www.researchgate.net/publication/220708287_GATE__a_Gen eral_Architecture_for_Text_Engineering

3. Grishman, R. (1986). Computational linguistics: an introduction. Cambridge University Press.

4. Ledeneva, Y., \&Sidorov, G. (2010). Recent advances in computational linguistics. Informatica, 34(1) Retrieved from: .https://www.informatica.si/index.php/informatica/article/view/2 $\underline{71 / 267}$

5. Rashel, Md. Mostafa (2011). Introducing Language Technology and Computational Linguistics in Bangladesh, International Journal of English Linguistics (IJEL), Vol. 1, No. 1, Pp- 179-186, March 2011, ISSN1925-869X, Canada. Retrieved

from http://www.ccsenet.org/journal/index.php/ijel/issue/view/321

\section{AUTHORS PROFILE}

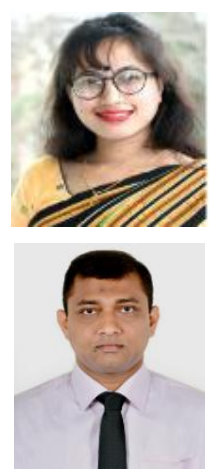

Puspanjali Hazarika is a research scholar of the Assamese language. She is interested in working in that field to develop the Assamese language in computational platforms. It is her first attempt in this field.

Md. Mostafa Rashel is an Assistant Professor of Linguistics in the Department of English at Daffodil International University, Bangladesh. Presently, Mr. Rashel is continuninghis $\mathrm{PhD}$ researcher in Linguistics at La Trobe University in Melbourne, Australia. His research interests include Endangered Indigenous Language Documentation and Description, Language Revitalization, Computational Linguistics INCL. Natural Language Processing (NLP). He has a number of publications on endangered languages of Chittagong Hill Tracts (CHT), Bangladesh. 